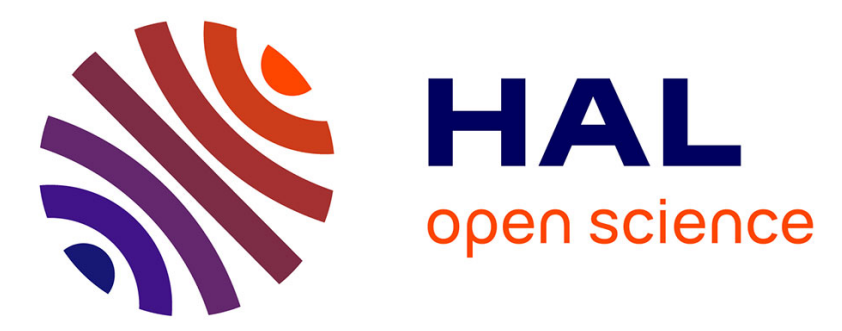

\title{
Damage assessment of pre-stressed structures: A SVD-based approach to deal with time-varying loading
}

Gianluca Ruocci, Gwendal Cumunel, Thi Thu Ha Le, Pierre Argoul, Nelly Point, Lamine Dieng

\section{To cite this version:}

Gianluca Ruocci, Gwendal Cumunel, Thi Thu Ha Le, Pierre Argoul, Nelly Point, et al.. Damage assessment of pre-stressed structures: A SVD-based approach to deal with time-varying loading. Mechanical Systems and Signal Processing, 2013, pp.In Press, Corrected Proof. hal-00880660

\section{HAL Id: hal-00880660 \\ https://hal-enpc.archives-ouvertes.fr/hal-00880660}

Submitted on 6 Nov 2013

HAL is a multi-disciplinary open access archive for the deposit and dissemination of scientific research documents, whether they are published or not. The documents may come from teaching and research institutions in France or abroad, or from public or private research centers.
L'archive ouverte pluridisciplinaire HAL, est destinée au dépôt et à la diffusion de documents scientifiques de niveau recherche, publiés ou non, émanant des établissements d'enseignement et de recherche français ou étrangers, des laboratoires publics ou privés. 


\title{
Damage assessment of pre-stressed structures: a SVD-based approach to deal with time-varying loading
}

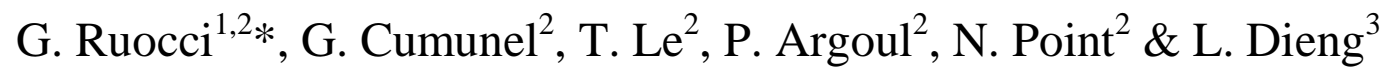 \\ ${ }^{1}$ Université Paris Est, IFSTTAR, MAT, F-75732 Paris Cedex 15, France \\ ${ }^{2}$ Université Paris Est, Laboratoire Navier (ENPC/IFSTTAR/CNRS), Ecole des Ponts ParisTech, $6 \& 8$ Avenue Blaise \\ Pascal, F-77455 Marne la Vallée Cedex 2, France \\ ${ }^{3}$ LUNAM Université, IFSTTAR, SOA, F-44341 Bouguenais, France
}

\begin{abstract}
Vibration-based methods are well-established and effective tools to assess the health state of civil, mechanical and aerospace engineering structures. However, their reliability is still affected by the variability of the features commonly used for damage detection. Environmental effects and changes in operational conditions are the main sources of variability in the structural response. As a consequence, the modal identification used to extract damage sensitive features has to face constricting requirements in terms of signals stationarity and performance accuracy. Moreover, with reference to the damage assessment, large variations of monitored features mask subtle effects due to damage, which remain undetected. This study is conceived to address both these issues by focusing, in particular, on the non-stationarity of the loading conditions of tensioned structures, such as cables and pre-stressed beams. The capability of spectral methods to deal with the modal identification of non-stationary systems is enhanced by a curve-fitting procedure based on nonlinear least squares optimisation. Wavelet analysis is applied for comparison and validation of the FFT-based technique. Identified natural frequencies are then used for the damage detection, exploiting the capacity of singular values decomposition to discriminate between damage-related events and the intrinsic non-stationary nature of the structural response. A reduced-order realization of the features set is performed to amplify changes not belonging to measurement variability but deriving from exogenous events, such as damage. The proposed methodology is validated by experimental analyses carried out on beams subjected to time-varying loading conditions in order to simulate the health monitoring of quasi and non-stationary systems.
\end{abstract}

KEYWORDS: pre-stressed structures, time-varying systems, non-stationary loading, damage detection, SVD, vibration measurements, experimental modal analysis, wavelet analysis.

\section{Introduction}

The maintenance and safeguard of civil structures and major bridges in particular are relying more and more on Structural Health Monitoring (SHM) systems. Representative examples of advanced applications of SHM systems on bridges all around the world are presented in [1-2]. The progressive technological

\footnotetext{
* Corresponding author. Tel.: +33619713314.

E-mail address: gianluca.ruocci@gmail.com (G. Ruocci)
} 
achievements have drastically reduced the constraints on the installation of permanent and widely distributed sensing networks that can allow measurement at very high sampling rates. Data acquisition and transmission do not represent any more an obstacle to vibration-based real-time health monitoring. However, data processing and information interpretation still require accurate investigations in order to adapt the diagnostic methods to the most recent measuring systems.

The evolution of environmental and operational conditions is the main challenge that monitoring systems have to face. Their effects on the structural response can be considerably higher than those due to damage events [3]. Changes of temperature and humidity, alterations in the structural mass or rigidity and variation of the boundary or loading conditions are the main sources of variability in the dynamic response of the structure. Two important consequences for the damage assessment can be drawn from this. First, the structure is considered as a time-varying system, implying that the extraction of damagesensitive features should be carried out accordingly. Secondly, the variability introduced by environmental and operational conditions acts as a mask that prevents a reliable estimation of the structural health state if its effects are not taken into account. A great effort has been made in recent years to develop methods that are able to discriminate between damage-related events and the intrinsic nonstationary nature of the structural behaviour.

Concerning the features extraction, a trade-off is required between the selection of quantities highly sensitive to damage and insensitive to external factors, and the requirement of their meaningfulness and exploitability within a continuous and fully-automated monitoring system. Thus, data processing must be oriented to estimate the modal properties of the structure even as they preserve a physical meaning that can be used to assess the estimation accuracy. Fourier spectral techniques [4] and experimental modal analysis methods, such as stochastic subspace identification [5], are commonly adopted to carry out this task. However, the extraction of modal properties through spectral and modal methods is restricted to linear systems and stationary processes. In the past, when the effect of the system response variability on the efficiency of damage assessment was acknowledged, time-frequency analysis was introduced to enhance the performance of Fourier transform. The Short-Time Fourier Transform (STFT) is the "natural" evolution of the Fourier analysis, since it simply consists in performing the spectral analysis on 
a limited time window and successively sliding the window along the time axis to get the time-frequency distribution.

A further enhancement in the trade-off between time and frequency resolution was achieved with the introduction of wavelet analysis. The wavelet transform can be interpreted as an adaptive window Fourier method [6], which employs local functions capable of handling non-stationary signals, providing a uniform resolution at all the scales. Although this appealing feature intrinsically entails the drawback of a poor resolution because of the limited size of the mother wavelet function, the technique remains one of the most successfully applied for the time-varying identification of modal properties [7-8]. Different methods, such as Time-Frequency (STFT, CWT) and time-variant SSI (Stochastic Subspace Identification) have been compared in [9] in reference to the case of a bridge crossed by moving load vehicles.

Recently, an innovative time-frequency method, known as Hilbert-Huang Transform (HHT), has been proposed by Huang et al. [10] to cope with non-stationary and non-linear time series. The method is the result of the coupled application of the Empirical Mode Decomposition (EMD) technique and the Hilbert Spectral Analysis (HSA). The EMD involves the extraction of the Intrinsic Mode Functions (IMFs) of a vibration signal. The IMF represents a simple oscillatory mode as a counterpart of a simple harmonic function. The mono-component nature of the IMFs allows the application of a well-behaved Hilbert Transform (HT) on each separated component in order to determine its instantaneous frequencies as well as other modal quantities. As an example, in [11] Yang et al. used the HHT to estimate the modal parameters of MDOF systems. Analogously to wavelet analysis, the modal properties extracted from the HHT can be selected as features in the damage detection procedure. In [12], Xu and Chen investigated the damage occurrence in the ASCE Benchmark, looking at the spikes introduced in the EMD by sudden changes in the structural stiffness, the natural frequencies and the damping ratios estimated using the HT. However, up to now very few contributions have been presented to validate the promising capabilities of this method on real-life structures subjected to significant environmental and operational variability.

Other time-frequency transformations are available to identify the modal properties of time-varying systems. Cohen's class time-frequency distributions, such as the Wigner-Ville Distribution (WVD), the Choi-Williams distribution (CWD), and the Volterra Series, are some of the methods usually applied to 
investigate the nonlinear behaviour of structures. However, so far the complex implementation and timeconsuming processing have prevented their adoption into the framework of continuous monitoring programs of real-life structures.

Eigen-properties and natural frequencies in particular, have proved to be significantly sensitive to the blurring effects of environmental and operational conditions and not directly exploitable as damage features in the assessment of engineering structures [13]. This issue represents a critical point for the reliable application of many vibration-based damage detection methods. However, some techniques relying on the decomposition of the monitored quantities according to orthogonal basis have shown promising capabilities in the distinction between environment and damage-related variabilities.

This paper is conceived to address the issues affecting the health monitoring of tensioned structures, such as cables and pre-stressed beams, when they are subjected to non-stationary loading conditions. First of all, a fundamental remark is made concerning the stationarity of the structural response in light of the monitoring purposes. Then, depending on the nature of the time-varying system, different identification techniques are used to track the evolution of the natural frequencies, which are successively employed as damage-sensitive features in the diagnostic process. A method based on the Singular Values Decomposition (SVD) of the feature trends is applied to discriminate two different sources of variability. The first source is protracted in time and related to changing environmental/operational conditions, while the second is introduced by the occurrence of an instantaneous damage event. This methodology is validated through the experimental analysis of a thin aluminium beam subjected to time-varying tensile forces, which is assumed representative of suspension bridge cables (typically short hangers). The capabilities of the proposed assessment method are tested by means of a comparison with a classical novelty detection algorithm. Some concluding remarks discuss possible strategies to integrate this diagnostic method in the framework of a real-time structural health monitoring program.

\section{Non-stationary system identification}

\subsection{A fundamental remark about time-variant monitored systems}

It is worthwhile to state a preliminary remark concerning the non-stationarity of the structural response of monitored systems. With reference to changing environmental and operational conditions that here are 
assumed responsible for the variability of the dynamic behaviour of structures, two possible situations can be distinguished. A system can be defined as either "quasi-stationary" or "non-stationary" depending on the relation between the changes of the mechanical system's response to time-variant excitations and the monitoring period and time-lag. In the first case, the structural state is assessed through short data acquisition time-lapses that are periodically repeated with a minimum delay. At the same time, the variation of the external factors is slow enough to consider the structural response as a steady state over the acquisition period.

This assumption leads one to discretize the variability of the structural response in a sequence of stationary states, i.e., a piece-wise stationary function, and allowing one to process them accordingly. This type of system is referred to as quasi or nearly stationary because, although varying in time, its components can be treated as time-invariant samples. This provides a remarkable simplification for the extraction of modal features, since it extends the applicability of data processing techniques to the whole range of methods that are based on the assumption of the stationarity of the acquired signals. If we consider the time length and delay commonly employed in the vibration acquisitions recorded by health monitoring systems of civil structures (i.e., a few minutes each hour), we can state that the majority of these applications can be considered quasi-stationary processes. However, some structures (mainly belonging to the mechanical engineering domain) require shorter and more frequent surveys to deal with the evolution of faster phenomena. Therefore, we refer to a non-stationary system when the changes of the environmental and operational conditions, and consequently those of the structural response, take place in a lapse of time comparable to the acquisition period. In this second case, the extraction of the modal features is restricted to time-frequency techniques. Both of the aforementioned situations are addressed in this work and some simple identification methods are used to extract modal quantities, adopted in the following as damage sensitive features, for the diagnosis of beam/cable-like structures.

\subsection{Modal features extraction based on Fourier spectral techniques}

When the dynamic behaviour of the structural system is assumed linear and stationary, or piece-wise stationary as in the present case, its modal parameters can be exhaustively estimated from the Frequency Response Function (FRF). In the case of a single-degree-of-freedom (SDOF) structure, modal damping 
ratios and natural frequencies are respectively related to the real and imaginary parts of the pole location of the FRF. The residue carries the absolute scaling of the FRF and is related to the corresponding mode shape. When working with relatively simple and lightly damped structures, such as cables and rectangular beams, the FRF can be reliably derived from measurements as the ratio of the output/input spectra. It will be different in the case of square cross-section beams with symmetrical boundary conditions due to the difficulty to identify coupled modes along two orthogonal bending directions. When modes are lightly coupled we can assume that the structure behaves as a SDOF around its modal frequencies. A technique known as Peak Picking [14] can be employed to extract a first estimate of the modal parameters. This technique consists in the selection of a certain number of modes depending on the quality of the measured signals, the complexity of the system and the expected sensitivity of the selected features to damage. For each mode, a frequency band is selected to isolate the peak in the FRF magnitude curve. The natural frequency of the mode corresponds to the coordinate of the FRF peak along the frequency axis, while the modal damping is determined by means of the half-power bandwidth method. This latter method consists in isolating each mode, and defines the $-3 \mathrm{~dB}$ bandwidth around the resonance peak. Equally, the modal damping $\zeta$ can be estimated using Eq. (1):

$$
\zeta=\frac{\Delta \omega}{\omega_{P}},
$$

where $\omega_{P}$ corresponds to the resonance frequency and $\Delta \omega$ is the bandwidth that is obtained as difference of the left and right-hand side frequency values by reducing the magnitude of the peak $A_{P}$ according to (2):

$$
A_{\Delta \omega}=\frac{A_{P}}{\sqrt{2}},
$$

with $A_{\Delta \omega}$ the value of the FRF whose corresponding frequency coordinates give the bandwidth.

For each mode, the natural frequency, modal damping ratio and resonance amplitude $A_{P}$ extracted from the measured FRF are employed as initial input parameters in an optimisation procedure aimed at fitting the experimental curve by means of an analytical representation. Under the assumption of a linear 
and proportionally damped structure, the FRF is defined as the function $H(\omega)$ of the three aforementioned modal parameters according to the following relationship:

$$
H(\omega)=\sum_{p} \frac{A_{p}}{\left(\omega_{p}^{2}-\omega^{2}+2 j \zeta_{p} \omega \omega_{p}\right)} .
$$

The functional defined as the difference between the measured and the analytically computed FRF is minimised by means of a non-linear least square algorithm in order to achieve an accurate estimate of the modal parameters. The curve-fitting is carried out over the frequency band resonance peak for each mode separately. The values of the three parameters resulting from the optimisation are retained as final estimates. This procedure allows reducing the bias in the modal parameters estimation due to the poor FRF resolution.

This method can be easily extended to the case of non-stationary systems. The key point consists in replacing the FRF with a Time-Frequency (TF) representation of the instantaneous energy spectrum $D(\omega, t)$. The functional to minimise becomes:

$$
D(\omega, t)-|H(\omega, t)|^{2}=0 .
$$

Inspired by the work of Ceravolo [15], a reasonable choice is the adoption of the Short-Time Fourier Transform (STFT), which corresponds to the Fourier Transform multiplied by a sliding time-window function. Although STFT is affected by the intrinsic limitations in the resolution of linear transforms, the simplicity of the technique provides a reasonable compromise between solution accuracy and computational requirements, as to deal with the problem in a direct manner. An important issue in the computation of the STFT concerns the type and number of samples of the time-window. The accurate selection of the time-window length has proved to influence the damping estimation to a large extent [15]. In the context of the following damage assessment, the features selection will be restricted to the natural frequencies, discarding modal damping because of its insensitivity to damage observed throughout the tests. However, some preliminary analyses were carried out to investigate the influence of the timewindow length on the estimation of the selected features.

The criterion used to define a first tentative value for the window length was a trade-off between the requirements of instantaneity of the estimate and that of completeness of the spectral response. In other 
words, the length of the time windowing must be limited to provide an estimate of the modal parameters sufficiently instantaneous to "finely discretize" the non-stationarity of the monitored system, but long enough to assure a frequency resolution that allows to distinguish harmonics components close in frequency. Nevertheless, the sampling frequency must be set taking the Nyquist rate into account in order to observe the dynamic response over the entire frequency spectrum of interest. Therefore, the selection of an appropriate compromise depends on the characteristics of the time-variant system and its excitation source. It would thus be difficult to provide a law of general validity. In the present case, this task was accomplished according to the indications provided in [15]. The selected value showed the substantial insensitivity of the estimated natural frequencies. Thus, the length of the time windowing was kept constant throughout the whole test and no adaptation was required.

The curve-fitting procedure adopted in the case of the locally stationary system was identically repeated for the strictly non-stationary system. More details about the application of both modal identification techniques to the case study of the beam subjected to time-variant stress are provided in the following sections.

The next step was to check and characterize the "level" of nonlinear effects. Time-frequency analysis is suitable for the analysis of nonlinear oscillations. In [16-17], the authors proposed several instantaneous functions based on the ridges and skeletons extracted from the wavelet analysis of free acceleration responses in order to characterize the behaviour of the structure. These instantaneous indicators do not require a particular model and can be considered as a characterization method. Their discrepancy from a linear case facilitates the detection and characterization of the non-linear behaviour of structures, allowing one to track the evolution of modal parameters as a time function. Their diagnosis approach offers the advantage of not requiring assumptions regarding the nature of the non-linearity analyzed. These indicators can be deduced from the computation of the Continuous Wavelet Transform (CWT) $W_{y}(b, a)$ of the studied signal $y(t)$ of finite energy:

$$
W_{y}(b, a)=\frac{1}{a} \int_{-\infty}^{+\infty} y(t) \psi^{*}\left(\frac{t-b}{a}\right) d t,
$$

where $\psi(t)$ is an analysing function called mother wavelet and $\psi^{*}(t)$ its complex conjugate. 
The parameters $a>0$ and $b \in \mathrm{R}$ vary continuously and introduce scale-dilation and time-translation, respectively. $a$ plays the role of the inverse of frequency and $b$ is related to time.

When the energy representation is based on the absolute value of the CWT $\left|W_{y}(b, a)\right|$, a ridge is a smooth and slow varying curve in the time-scale plane defined by $\left(b, a_{r}(b)\right)$ and where $\left|W_{y}(b, a)\right|$ is locally maximum. The absolute value of the CWT along the ridge $\left|W_{y}\left(b, a_{r}(b)\right)\right|$ is called the skeleton. The ridges can be extracted from the maxima of $\left|W_{y}(b, a)\right|$ (see $[7,16,18]$ for more details).

The first indicator $I_{1}(b)$ depends on time $b$ and is defined for each ridge $a_{r}(b)$ within the CWT of the signal by (see. [16-18]):

$$
I_{1}(b)=\frac{1}{2 \pi} \frac{\omega_{0}}{a_{r}(b)},
$$

where $\omega_{0}$ is the angular frequency where $|\Psi(\omega)|$ is maximum, $|\Psi(\omega)|$ being the Fourier transform of the mother wavelet $\psi(t): \Psi(\omega)=\int_{-\infty}^{+\infty} \psi(t) e^{-i \omega t} d t$, where $i=\sqrt{-1}$.

Let us study the case where $y(t)$ is a chirp, a signal of type $A(t) \cos (\phi(t))$, in which the frequency increases or decreases with time, and the time variation of the amplitude $A(t)$ is slow compared to that of the phase $\phi(t)$ (this signal is then described as asymptotic see. [16-18]). In that case, the energy tends to "localize" around the ridge (see. [18]):

$a_{r}(b)=\frac{\omega_{0}}{\phi^{\prime}(b)}$,

where $\phi^{\prime}(b)=\frac{d}{d b} \phi(b)$,

and the CWT of $y(t)$ is given by:

$$
W_{y}(b, a) \approx \frac{1}{2} A(b) e^{i \phi(b)} \overline{\Psi\left(a \phi^{\prime}(b)\right)} .
$$

The indicator function $I_{1}(b)$ is then equal to:

$$
I_{1}(b)=\frac{1}{2 \pi} \phi^{\prime}(b)
$$


It characterizes an instantaneous frequency and has the unit of a frequency $(\mathrm{Hz})$. In the particular case of free acceleration responses $y(t)=y_{k}(t)=\sum_{j=1}^{n} y_{k j}(t)$ of discrete linear mechanical systems at point $k$, $I_{1}(b)$ can be rewritten for the $\mathrm{j}^{\text {th }}$ mode, by:

$$
I_{1 j}(b)=\frac{1}{2 \pi} \omega_{d j}=\frac{1}{2 \pi} \omega_{j} \sqrt{1-\xi_{j}^{2}},
$$

where $\omega_{j}$ and $\xi_{j}$ are respectively the angular frequency for the conservative underlying system and the modal damping ratio for the $\mathrm{j}^{\text {th }}$ mode (see [17-18] for more details).

The resolution of the CWT is a relevant criterion especially in the procedure of detecting and characterizing nonlinear systems. To choose the mother wavelet resolution, the authors introduced the Qfactor $[7,16]$, defined as the ratio between the centre-frequency and the frequency bandwidth of the mother wavelet. More precisely, in [17], three reference values of $Q$ are defined to address edge effect problems and modal coupling, namely: $Q_{\min }, Q_{\max }$ and $Q_{\xi}$. Their expressions are respectively, $Q_{\min }=2.5 \frac{\omega_{j}}{d \omega_{j}}, Q_{\max }=0.1 \omega_{j} L$ and $Q_{\xi}=\frac{1}{\sqrt{2} \xi}$, where $L$ is the duration of $y(t), \omega_{j}$ is the analyzed angular frequency, and $d \omega_{j}$ is a characteristic discrepancy between two close frequencies (see [7, 17] for more details). The inequality $Q_{\min }<Q_{\max }$ must always hold and two cases are distinguished: (1) if $Q_{\min }<Q_{\xi}$, then $Q=\min \left(Q_{\xi},\left(Q_{\min }+Q_{\max }\right) / 2\right)$; and (2) if $Q_{\text {min }} \geq Q_{\xi}$, then $Q=Q_{\min }$. In this study, the Cauchy's mother wavelet is used: $\psi(t)=\psi_{n}(t)=\left(\frac{i}{t+i}\right)^{n+1}$, which is admissible and progressive. Given we have $Q=\frac{\sqrt{2 n+1}}{2}$ and $\omega_{0}=n$, its Fourier transform is $\Psi(\omega)=\frac{2 \pi}{n !} \omega^{n} e^{-\omega} \Theta(\omega)$, where $\Theta(\omega)$ is the unit step function and $\Psi\left(\omega_{0}\right)=2 \pi \frac{n^{n}}{n !} e^{-n}$.

Finally, the $I_{1}(b)$ indicator will allow us to characterize the degree of nonlinear effects in the system's responses. To do that, the variation coefficient defined by $\sigma_{f} / \bar{f}$ is computed, where $\sigma_{f}$ and 
$\bar{f}$ are respectively the standard deviation and the mean value of $I_{1}(b)$ within the domain where the edge effects can be neglected.

\section{The experimental case study}

\subsection{The problem of varying loading conditions in pre-stressed structures}

Pre-stressed elements are differently employed in many civil engineering structures, such as in stay cable bridges, suspension bridges and pre-stressed concrete bridges. Cables in particular are critical structural components because of the important bearing functions they accomplish and their vulnerability to environmental phenomena. Stay cables, suspensions and hangers transfer deck loads to piers, while in pre-stressed concrete bridges cables are used to fit box girders. Cables in bridges are mainly subjected to two kinds of damage mechanisms: i) corrosion due to aggressive marine environment, rain, snow and anti-icing salts; ii) fretting fatigue phenomena due to cyclic traffic loads and wind action [19]. Beyond thermal and operational (traffic) effects, cable oscillations produced by wind and/or rain are also dangerous. Indeed, these oscillations can potentially induce cyclic stresses that lead to fatigue phenomena in the stays themselves as well as in the anchorages. Karman whirlwind vortexes have been observed to generate high-frequency vertical modes on stay cables and long suspension cables (hangers) [20]. When wind is combined with rain, water rivulets appear on the two opposite sides of the cable, potentially causing lateral oscillations whose frequencies are lower than those of the Karman phenomena.

The most frequent damage events in stay cables are wire ruptures induced by corrosion and/or fatigue. These two phenomena result often in a local loss of mass, affecting either the total mass or the bending stiffness of the cable. Therefore, it is reasonable to address the problem of damage detection, location and quantification by using observed changes of dynamic characteristics, for instance FRF or modal parameters, as sensitive indicators of the structural integrity. However, structural disturbance detection in bridge stay cables using experimental vibration data is a particularly hard task due to the variability of the loading conditions. Indeed, we have already stressed that modal parameters are extremely sensitive to environmental/operational conditions, whose changes can mask more subtle variations induced by damage. Tensile stress may vary considerably as a consequence of traffic, temperature and wind cycles. It 
is worthwhile to notice that those sources of variability may act separately or together and over different time-scales. Thus, the variation of loading conditions may assume complex trends which are difficult to define even from the correlation with in-situ measurements of environmental variables. Moreover, depending on the rapidity with which the loads change, the health monitoring has to deal with a timevariant system characterized by different degrees of non-stationarity.

\subsection{Description of the laboratory case study}

Two aluminium pre-stressed beams were chosen for the experiments because they are more practical to test compared to cables, yet show similar structural behaviour. One beam is used for the quasistationary test and the other for the non-stationary test. The beams are $520 \mathrm{~mm}$ long, $25 \mathrm{~mm}$ wide and 2 $m m$ thick. The extremities are clamped by the wedge grips of a universal testing machine INSTRON 6022 over a length of $40 \mathrm{~mm}$. The useful length of the beams thus measured $L=440 \mathrm{~mm}$ and is instrumented with 10 accelerometers BK-4507 (see Fig. 1), regularly spaced every $40 \mathrm{~mm}$. The sensors signals are digitalized by NI 9234 CompactDAQ modules with a sampling frequency of $4000 \mathrm{~Hz}$.

A dimensionless parameter $\zeta=\sqrt{\frac{E I}{T L^{2}}}$ is often introduced [21-22] in order to characterize the importance of the tension compared to the flexural rigidity of the beam. Some authors proposed bounds for $\zeta$ allowing to distinguish between three behaviours: vibrating string, beam without tension or prestressed beam. When the value of $\zeta$ is weak enough: $\zeta<0.002$ [22], the bending does not have a significant effect on the low natural frequencies. The structure behaves close to a vibrating string and its natural frequencies evolve rather linearly with respect to the mode number, $f_{k} \approx k \cdot f_{l}$. Conversely, when the value of $\zeta$ is high enough: $\zeta>2$ [22], the bending stiffness is predominant and the Eigen frequencies increase with the square of the mode number, $f_{k} \approx k^{2} \cdot f_{1}$. When the tension tends towards $0, \zeta$ tends to infinity. The structure behaves close to an Euler Bernoulli beam without tension. Finally, within these bounds: $0.002 \leq \zeta \leq 2$, the structure behaves like a pre-stressed beam.

The following values have been considered for the mechanical and geometrical parameters of the aluminium beams: Young modulus: $E=69 \cdot 10^{9} \mathrm{~Pa}$, tension $T=2000 \mathrm{~N}$, area moment of inertia $I=$ $1.67 \cdot 10^{-11} \mathrm{~m}^{4}$, mass per unit length $\mu=0.135 \mathrm{Kg} / \mathrm{m}$. The dimensionless parameter that we obtain is 
$\zeta=0.0545$. Thus, the structure behaves like a pre-stressed beam but the effect of the flexural rigidity is weak.
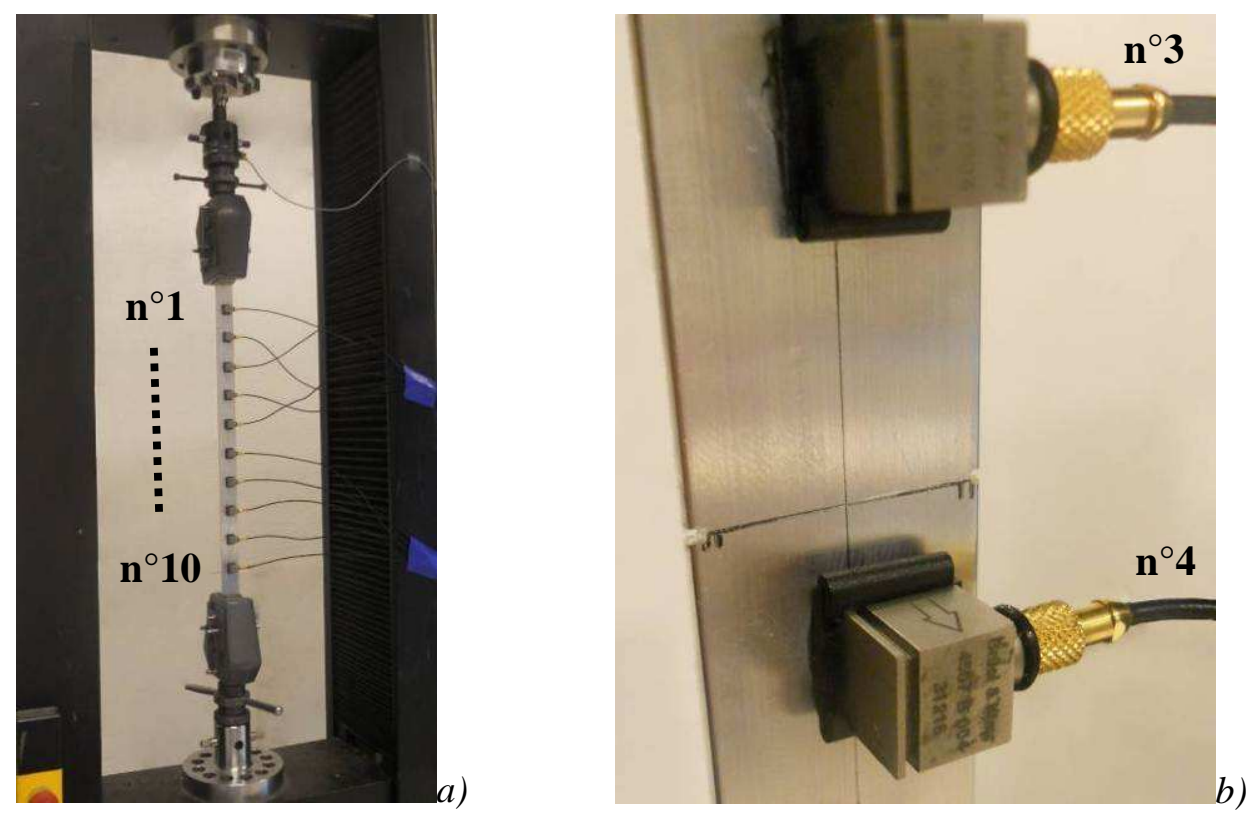

Figure 1: Photographs of the instrumented pre-stressed beam (a) and the damaged zone (b).

For the quasi-stationary test, 300 load cases are applied before introducing damage by saw-cut between accelerometers $\mathrm{n}^{\circ} 3$ and 4 . The saw-cut is $1 \mathrm{~mm}$ wide and $1 \mathrm{~mm}$ deep on each side of the beam (see Fig. 1). The load cases (see Fig. 2) followed the stress law defined in Eq. (11):

$$
T(t)=T_{0} \cdot\left[e^{-\alpha t}+\frac{1}{\beta \cdot \cos (\varphi)} \cdot \cos \left(\omega_{0} t \sqrt{1-\delta^{2}}-\varphi\right) \cdot e^{-\delta \omega_{0} t}\right]
$$

where $T_{0}$ is the pre-stress force at $t=t_{0}, \alpha$ is the long-term damping coefficient, $\beta$ is a constant relating the oscillation amplitude to the initial force value $T_{0}$ (here selected equal to 30 ), $\varphi=\arctan \left(\delta / \sqrt{1-\delta^{2}}\right), \omega_{0}$ is the pulsation of the perturbation and $\delta$ is the short-term damping coefficient. The initial pre-stress force $T_{0}$ is $2.0 \mathrm{kN}$, which makes $T$ at $t=t_{0}$ equal to $2.06 \mathrm{kN}$ and decreasing to $1.96 \mathrm{kN}$ at the end of the undamaged stage. A Gaussian random noise with mean equal to the initial pre-stress force $T_{0}$ and 0.5 of standard deviation was added to the stress law given by Eq. (11). The average ratio between the noise and the stress law from Eq. (11) is close to 5\%. The resulting loading history is applied to the beam in order to simulate a complex load variation, obtained by the superposition 
of phenomena acting over different time-scales (for example, the combination of daily and seasonal variations related to thermal effects).

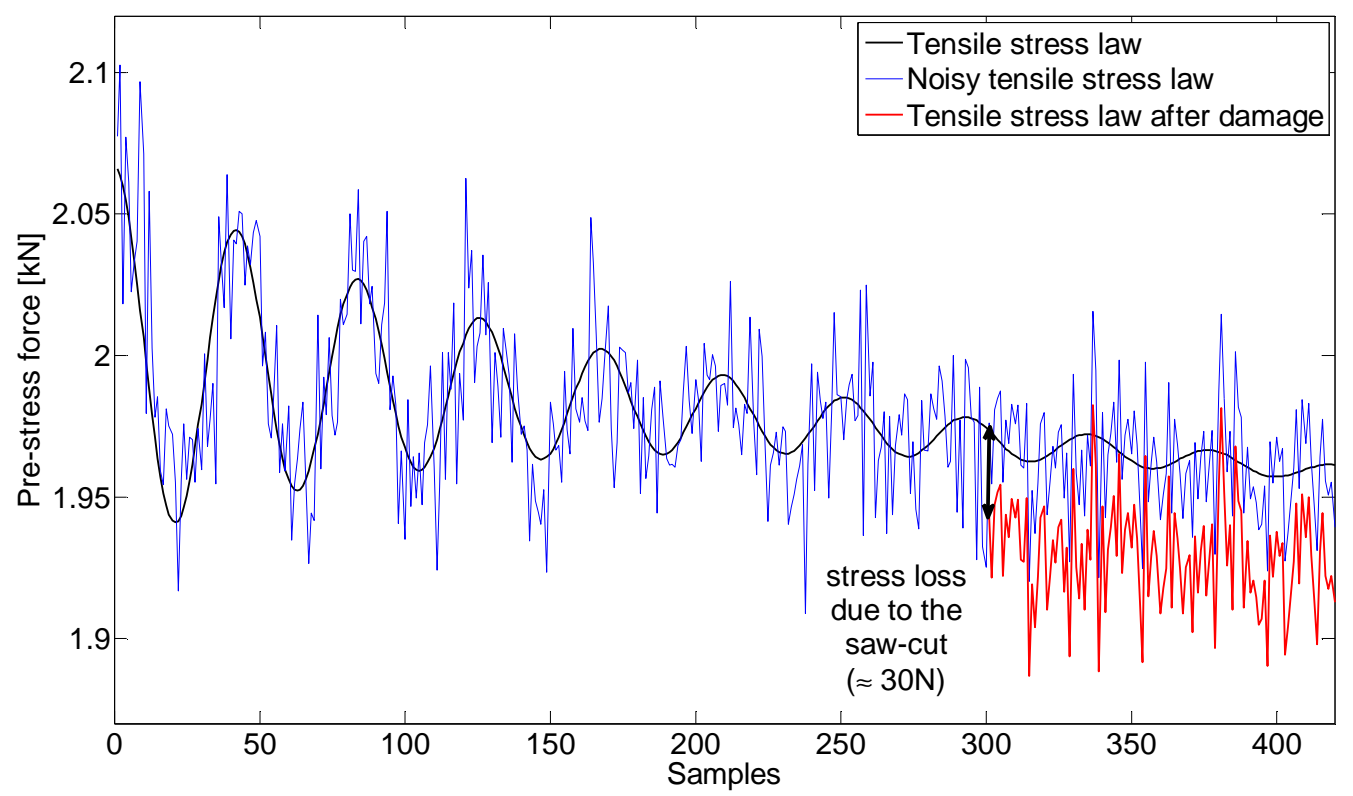

Figure 2: Pre-stress force applied to the beam.

At each load case, stress is kept constant and about ten shocks, located between sensors n ${ }^{\circ} 8$ and 9 , are performed with an impact hammer BK-8206 over a 30s period. When damaging the beam by the saw-cut, the movable crosshead of the testing machine is held in position. A stress loss is observed because the applied load is forced to decrease to maintain the equilibrium. This loss, of about $30 \mathrm{~N}$, is measured by the load cell of the testing machine and is subtracted from the stress law of the following load steps. Then, 120 samplings are performed in a similar manner on the damaged structure.

For the non-stationary test, the stress law is the same as before, as are the characteristics of the saw-cut and the sampling frequency. In this case, a white noise is added by a modal exciter BK-4810 suspended to the testing machine frame and connected to the beam by a stinger located between sensor $n^{\circ} 8$ and 9 . The exciting force is measured by an impedance head BK- 8001 added on the shaker. The total duration of the test is ten minutes and the movable crosshead of the testing machine is controlled by force in order to follow the imposed stress law.

\subsection{Results of the modal identification}

\subsubsection{Quasi-stationary test}


Once the vibration signatures are acquired, a common pre-processing stage is carried out for both the beams. Since the analysis is restricted to the identification of the first four natural frequencies, the spectrum of interest is bounded to 60 to $800 \mathrm{~Hz}$ while the other spectral components are filtered out in order to prevent the selection of spurious peaks. The number of identified modes is limited in order to improve the accuracy of damage detection methods in realistic and constraining conditions. When dealing with cables and beam-like structures used in civil engineering, only a limited number of modes are accurately identified and available for damage assessment purposes. All the sensors deployed on the beams are used in the modal parameters estimation in order to achieve a better mode shape resolution. Concerning the natural frequencies, the analysis of reduced sets of sensors shows a substantial insensitivity for the identification results. A number of equally-spaced sensors, varying from 3 to 4 , provides a good characterisation of the dynamic behaviour of the beam over the selected spectrum. Moreover, the spectral responses derived from each sensor are averaged to enhance the capability of detecting all the resonance peaks.

The acquired signals are then subdivided or merged according to the non-stationarity of the analysed system. In the case of the "quasi-stationary" beam, the free-decay response to each hammer shock is selected and treated separately. The modal parameters are identified according to the explanations provided in Section 2.2, and only the results derived from the most excited response (i.e., the highest peak) are retained for each tensile force step. Conversely, the continuous acquisition of the non-stationary response of the second beam required a sequential recording to limit the size of the data. Each data block is analysed separately and the results are merged to obtain a continuous evolution of the modal features throughout the time of the test.

The FRFs of the piece-wise stationary beam are estimated using the Welch method with 4096 samples of resolution to compute the FFT. The curve-fitting, repeated for the selected shocks of all the acquired stress steps, provides the time evolution of the optimal values of the modal parameters that are used to define the $H(\omega)$ function in (3). The natural frequencies of the first four modes are depicted in Fig. 3. The cyclic variations of the frequencies due to the time-variant loading conditions are clearly recognizable, whereas the damage occurrence at the $300^{\text {th }}$ step is difficult to detect at sight. 

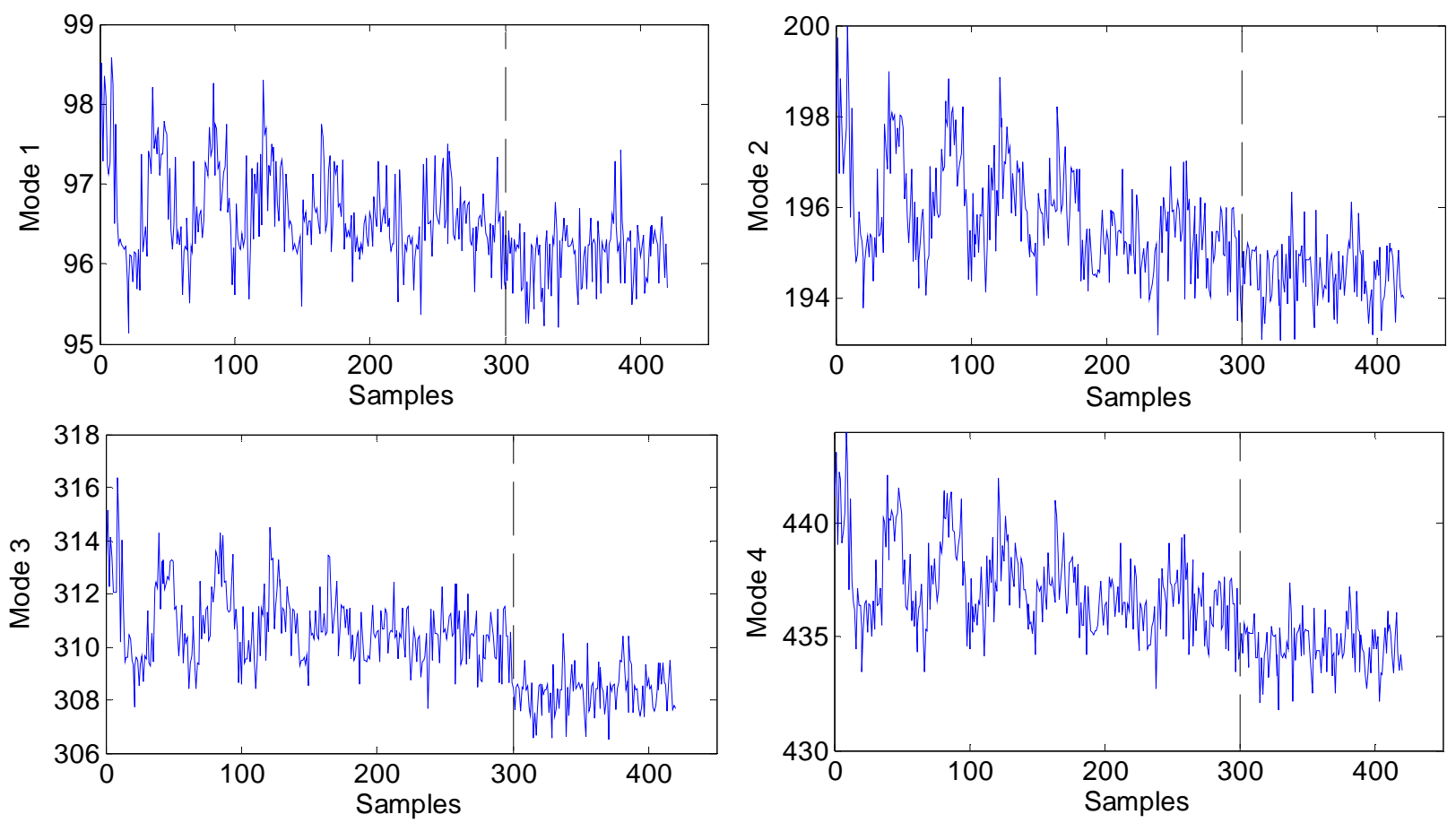

Figure 3: Evolution of the natural frequencies $[\mathrm{Hz}]$ throughout the tests carried out on the quasi-stationary system: damage introduced by saw-cut after the $300^{\text {th }}$ sample (dashed vertical line).

A high accuracy in the identification of the mode shapes was also observed. For the sake of clarity, only the averaged mode shapes for the healthy and damaged system are presented in Fig. 4. No significant distinctions between the two states are detectable. The mode shapes are not considered as damage features in this work since the aim here is limited to the simple detection of the damage occurrence. However, some numerical analyses show promising results for the capability of mode shapes to extend the proposed method to damage location purposes. On the contrary, as expected, the damping ratios appeared insensitive to damage and were substantially invariant throughout the tests. The absence of friction and fatigue cracks justifies the experimental result. Therefore, damping is not considered as a damage feature in the assessment process.

The natural frequencies of the first four modes obtained by FRF-fitting are compared with the results of the CWT method [7]. For the sake of brevity, Table 1 presents the results of the two methods for only three load cases of the healthy state. Similar results have been found for the other load cases. Table 2 presents the choice of Q-factor for each mode, used to decide the mother wavelet resolution. 

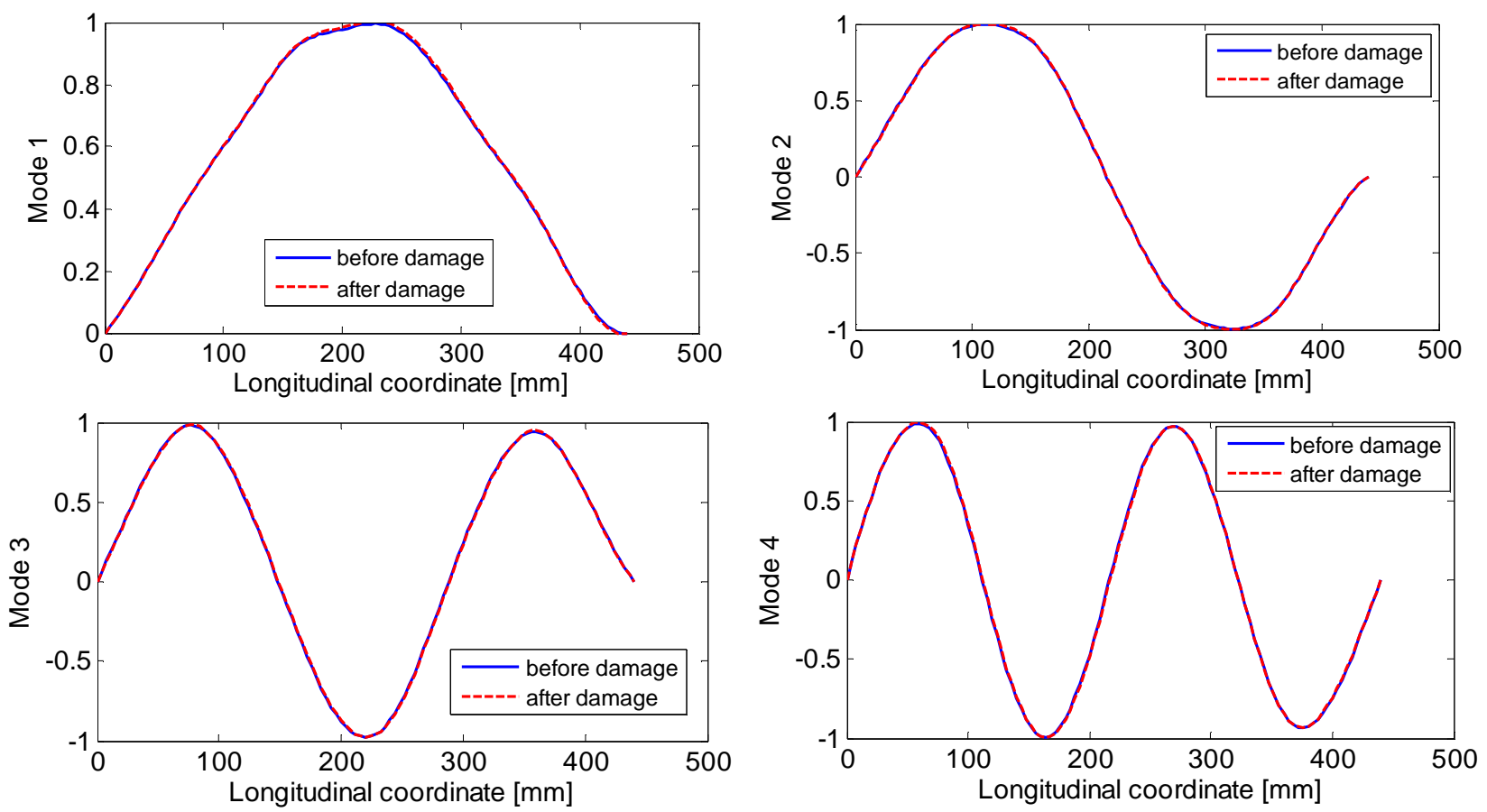

Figure 4: Averaged mode shapes of the healthy (solid blue line) and damaged (dashed red line) states for the quasistationary system.

Fig. 5 depicts the first indicator $I_{1}$, defined in Eq. (6), of the first four modes of the beam, obtained for the $2080 \mathrm{~N}$ stress load case. As the first indicator $I_{1}$ is not a straight line, we can conclude that frequency is changing in time and that the system shows a nonlinear behaviour. However, the very low values (the average is below 0.5 ) of the variation coefficient, defined by the ratio of the standard deviation to the mean of $I_{1}\left(\sigma / \overline{\mathrm{I}}_{1}\right)$, shown in Table 1 indicate that the nonlinearity is weak, thus justifying the adoption of FFT-based methods. Besides, a limited relative variation is also observed between natural frequencies obtained by FRF-fitting and CWT methods. As the dimensionless parameter $\zeta=0.0545$ is low, the effect of the flexural rigidity is weak and the ratio of the frequencies to the first one, $f_{n} / f_{1}$ (in brackets in the third column of Table 1), is closer to the case of cables than beams. 
Table 1. Comparison between natural frequencies estimated by means of FRF-fitting and CWT method for the quasi-stationary system and different levels of axial pre-stress.

\begin{tabular}{|c|c|c|c|c|c|}
\hline \multirow[b]{2}{*}{ Load $[\mathrm{N}]$} & \multirow[b]{2}{*}{ Mode } & \multicolumn{4}{|c|}{ Frequency [Hz] } \\
\hline & & $\begin{array}{l}\text { FRF-fitting } \\
\left(\text { ratio } \mathrm{f}_{\mathrm{n}} / \mathrm{f}_{1}\right)\end{array}$ & $\begin{array}{l}\text { CWT method } \\
\text { (mean value) }\end{array}$ & $\begin{array}{l}\text { CWT method } \\
\left(\sigma / \overline{\mathrm{I}}_{1}\right)[\%]\end{array}$ & $\begin{array}{c}\text { Relative variation } \\
\text { (FRF/CWT) } \\
{[\%]}\end{array}$ \\
\hline \multirow{4}{*}{2018} & 1 & $97.29(1)$ & 97.73 & 0.04 & 0.45 \\
\hline & 2 & $196.75(2.02)$ & 196.11 & 0.51 & 0.33 \\
\hline & 3 & $312.27(3.21)$ & 313.08 & 0.12 & 0.26 \\
\hline & 4 & $439.06(4.51)$ & 440.74 & 0.18 & 0.38 \\
\hline \multirow{4}{*}{2056} & 1 & $98.11(1)$ & 98.29 & 0.04 & 0.18 \\
\hline & 2 & $197.76(2.02)$ & 197.41 & 0.68 & 0.18 \\
\hline & 3 & 313.38 (3.19) & 314.22 & 0.15 & 0.27 \\
\hline & 4 & $441.91(4.5)$ & 443.12 & 0.23 & 0.27 \\
\hline \multirow{4}{*}{2080} & 1 & $98.36(1)$ & 98.59 & 0.13 & 0.23 \\
\hline & 2 & $198.83(2.02)$ & 198.21 & 0.56 & 0.31 \\
\hline & 3 & $314.12(3.19)$ & 315.43 & 0.12 & 0.42 \\
\hline & 4 & $442.23(4.5)$ & 443.77 & 0.13 & 0.35 \\
\hline
\end{tabular}

Table 2. CWT method Q-factor chosen for each mode.

\begin{tabular}{ccccc}
\hline & Mode 1 & Mode 2 & Mode 3 & Mode 4 \\
\hline$Q_{\min }$ & 3 & 5 & 7 & 9 \\
$Q_{\max }$ & 93 & 187 & 99 & 139 \\
$\xi$ & $0.78 \%$ & $0.74 \%$ & $0.88 \%$ & $0.85 \%$ \\
$Q_{\xi}=1 /(\sqrt{2} \xi)$ & 91 & 96 & 80 & 83 \\
$Q=\left(Q_{\text {min }}+Q_{\max }\right) / 2$ & 48 & 96 & 53 & 74 \\
\hline
\end{tabular}

The same indicator $I_{1}$ is portrayed in Fig. 6 for the first four modes of the beam in its damaged state. Load cases and shocks for each mode are selected in order to obtain the same amplitude for the excitation force as in the previous case, and so to allow a comparison. The result in terms of strength of the nonlinearity is not far from that of the undamaged state. A nonlinear behaviour is still detected, but damage seemed not to increase significantly. The shifts in the natural frequencies are comparable to those observed in Fig. 3 for the FRF-fitting technique, proving the accuracy of the latter. Therefore, for the sake of simplicity, the FRF-fitting is chosen as the features extraction method for the following damage assessment because of its rapidity in the processing of large amounts of experimental data. 

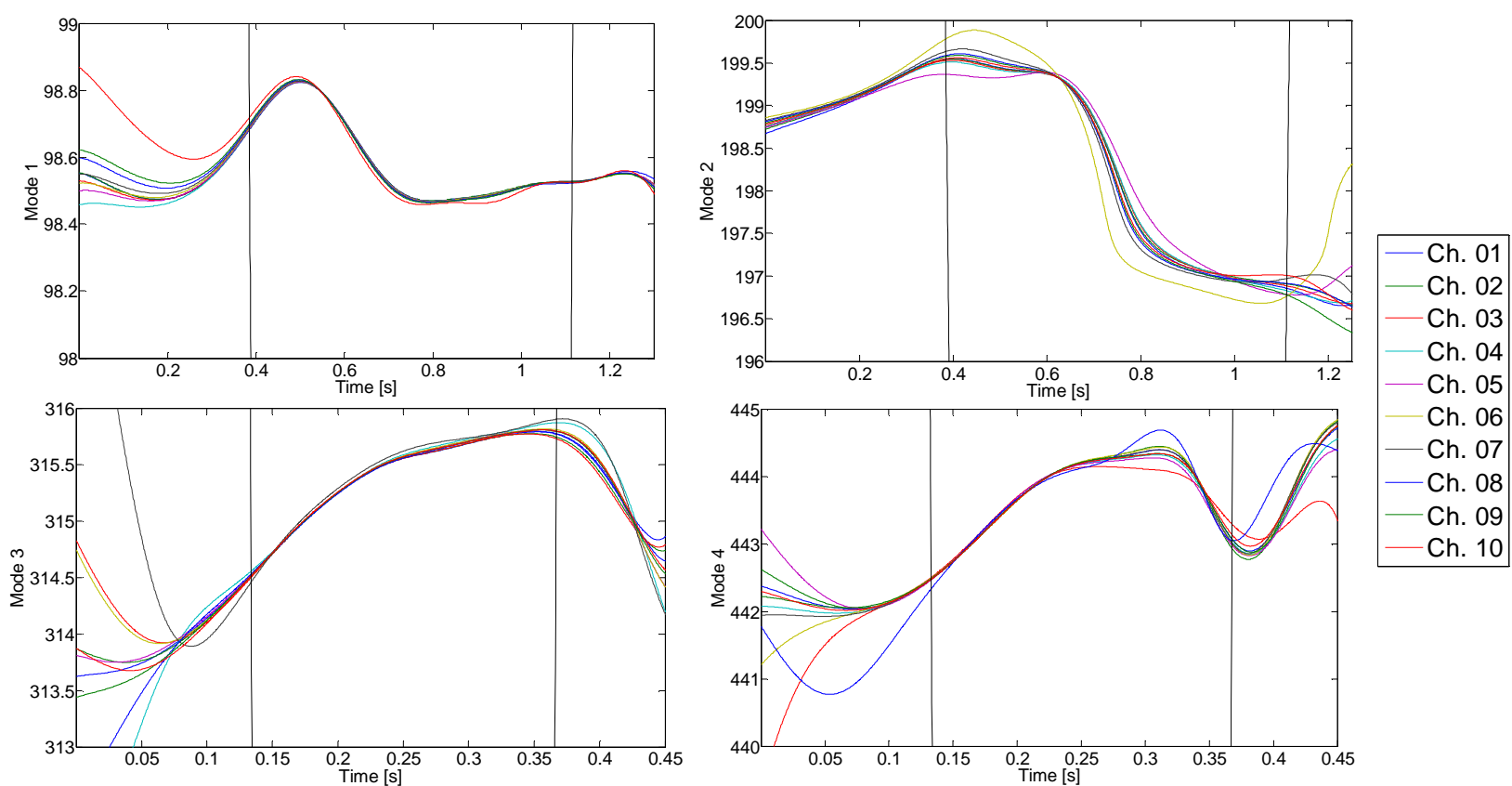

Figure 5: First indicator $I_{1}$ obtained for the first four modes of the beam in the undamaged configuration.
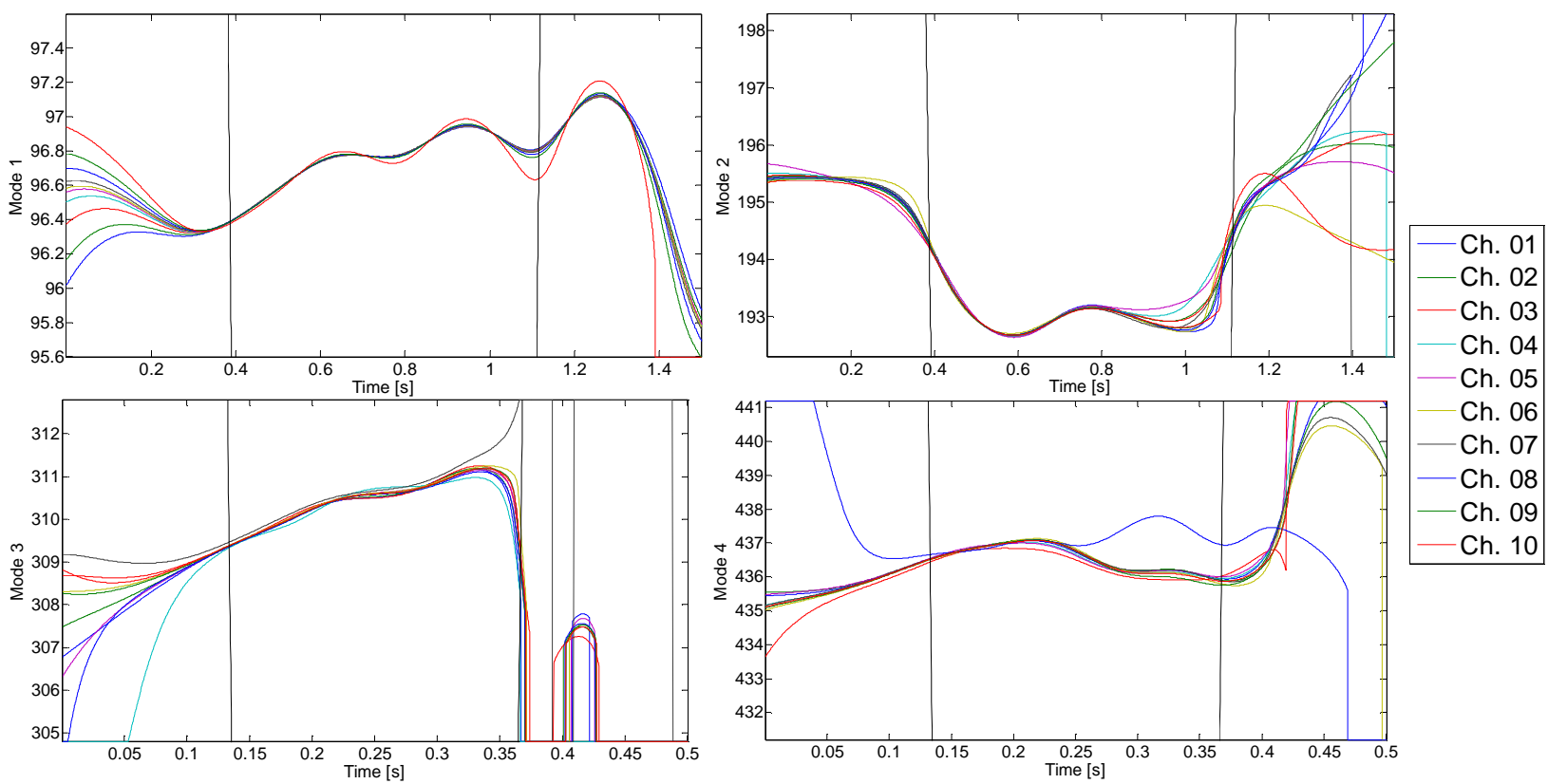

Figure 6: First indicator $I_{1}$ obtained for the first four modes of the beam in the damaged configuration.

In [22], changes of intrinsic characteristics of the beam due to local "small" reduction or increase of flexural rigidity and weight in a localized area of a pre-stressed beam combined or not with a global variation of the tension have been studied. Local change of the bending stiffness is denoted $\Delta E I$ and is assumed to be zero outside the interval $\left[x_{0}-\frac{\Delta L}{2}, x_{0}+\frac{\Delta L}{2}\right]$. The variation of the tension is assumed to 
be constant along the beam and denoted by $\Delta T$. It is also assumed to have no change in the mass density. A first order approximation of the change of the Eigen frequencies due to these small variations can then been obtained by neglecting the variations of order 2 :

$$
\frac{\Delta f_{n}}{f_{n}} \approx v_{n} \frac{\Delta T}{T}+\tau_{n} \frac{\Delta E I}{E I} \frac{\Delta L}{L}\left(\varphi_{n}^{\prime \prime}\left(x_{0}\right)\right)^{2}
$$

where $v_{n}=\frac{1}{2\left(1+\zeta^{2} L^{2} \frac{\left\|\varphi_{n}^{\prime \prime}\right\|_{2}^{2}}{\left\|\varphi_{n}^{\prime}\right\|_{2}^{2}}\right)}, \tau_{n}=\frac{\zeta^{2} L^{3}}{2\left(1+\zeta^{2} L^{2} \frac{\left\|\varphi_{n}^{\prime \prime}\right\|_{2}^{2}}{\left\|\varphi_{n}^{\prime}\right\|_{2}^{2}}\right)} \frac{1}{\left\|\varphi_{n}^{\prime}\right\|_{2}^{2}}$ with $\varphi_{n}(x)$ is the n-th normal mode of unit norm according to $\mathrm{L}^{2}[0, \mathrm{~L}]:\left\|\varphi_{n}\right\|_{2}^{2}=\int_{0}^{L} \varphi_{n}^{2}(x) d x=1, \varphi_{n}^{\prime}(x)$ and $\varphi_{n}^{\prime \prime}(x)$ being the first and the second derivative, respectively.

Fig. 7 gives for the four first modes $(1 \leq n \leq 4)$, the evolution throughout the tests carried out on the quasi-stationary system of $\frac{\Delta f_{n}}{f_{n}}$ (blue curve) and of $v_{n} \frac{\Delta T}{T}$ (red curve), where $\Delta f_{n}=\frac{f_{n}(t)-\bar{f}_{n}}{\bar{f}_{n}}$ and $\frac{\Delta T}{\bar{T}}=\frac{T(t)-\bar{T}}{\bar{T}}$ with $\bar{f}_{n}$ and $\bar{T}$ the mean value of the n-th frequency and the tension, respectively for the 299 tests before damage (saw-cut). It can be noted that after the damage, for the two first modes, a difference between the red and the blue curves is clearly visible. This difference may depend on the damage position $x_{0}$ (cf. Eq. (12)). Further investigations of this difference are under study in order to detect the spatial localisation of the damage. 

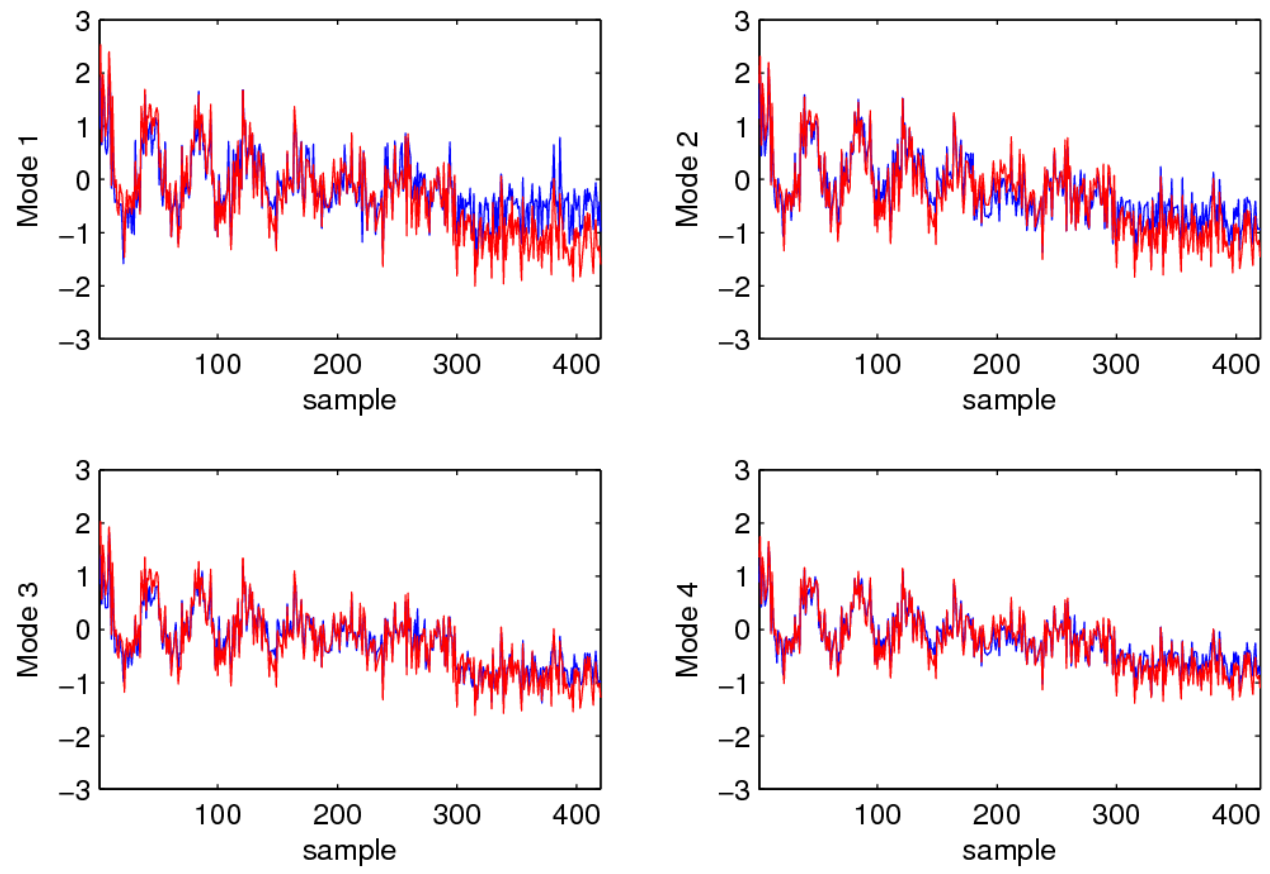

Figure 7: Evolution of $\frac{\Delta f_{n}}{f_{n}}$ (blue curve) and of $v_{n} \frac{\Delta T}{T}$ (red curve) throughout the tests carried out on the nonstationary system for the four first modes. Damage is introduced by saw-cut after the $300^{\text {th }}$ sample.

\subsubsection{Non-stationary test}

In the estimation of the modal parameters of the time-variant beam, preliminary signals decimation is performed in order to accelerate the computation. A Hanning-type window with a length of 80 samples, and with an overlap of 2 samples, provides an optimal STFT representation. The set of initial values for the three variables used to start the optimisation is derived from the first estimate of the previous case study. The minimisation of Eq. (4) is repeated at each time-step and the initial values of the modal parameters are constantly updated with the results of the previous calibration. Finally, a moving average is applied to smooth the instantaneous evolution and minimize spurious peaks. By way of example, the STFT of a 10s-long signal filtered over the frequency band of the first four modes is depicted in Fig. 8, together with the result of the moving averaging on the curve-fitting outcome. 


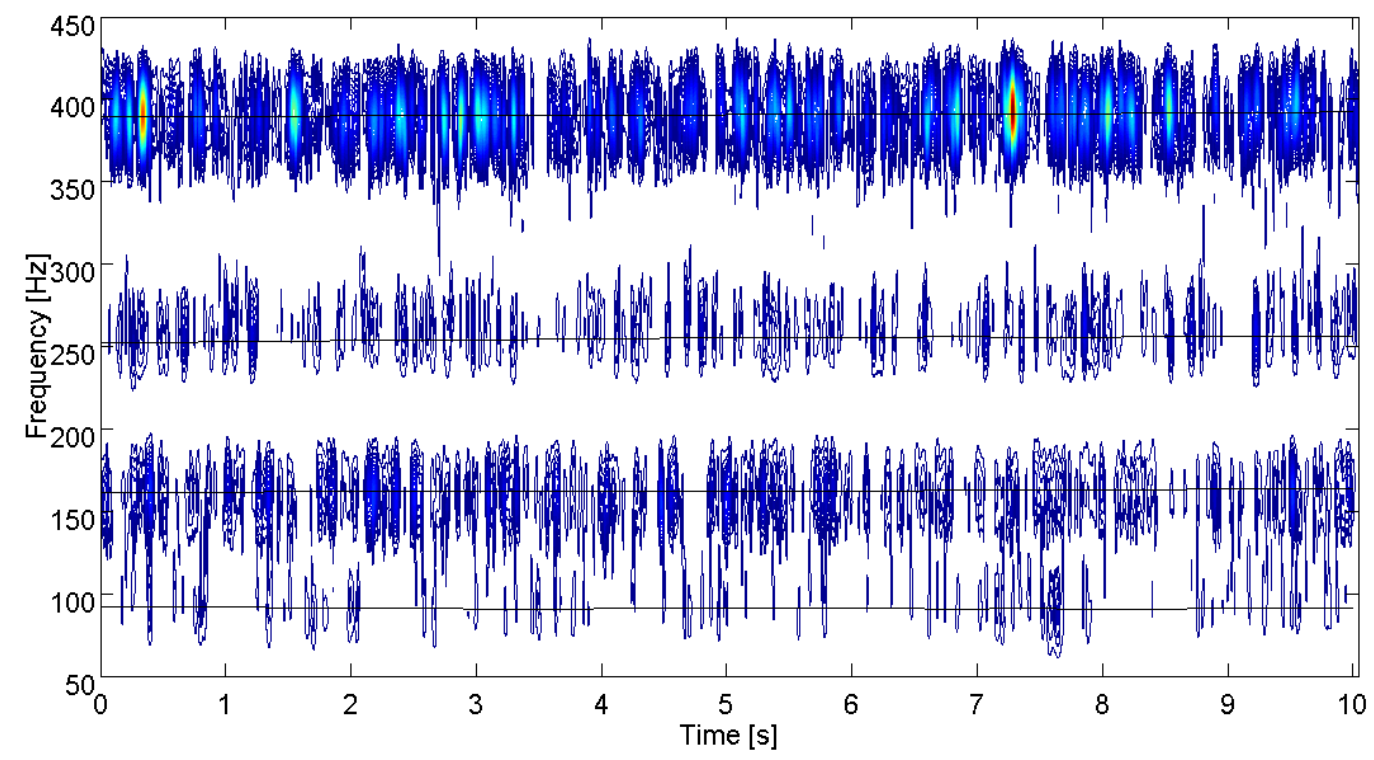

Figure 8: Time-frequency distribution from the STFT applied on a 10s-long sample acquired by sensor $n^{\circ} 5$ for the undamaged state of the non-stationary system: the moving average smoothing on the curve-fitting result is depicted by black solid lines.

Fig. 9 shows the averaged results obtained from the time-frequency analysis of the recorded blocks of signals for the healthy and damaged beams, after these data were merged together. The modal frequency estimates turn out to be quite satisfactory: with the exception of the first mode, the influence of the loading conditions is clearly identifiable by the cyclic evolution mainly focused at the beginning of the test. However, a large drop in the frequency is obtained for the last two modes. This result is explained by the perturbation of the connection between the modal exciter and the beam at the instant of the saw-cut. Indeed, the shaker is located at about $80 \mathrm{~cm}$ from one edge of the beam, close to the anti-nodes of the third and fourth modes. Therefore, the capability of the SVD to distinguish damage events from varying loading conditions will be verified only with the results of the previous time-variant identification, since this provides a more challenging task to accomplish. A final remark concerning the shift of the estimated frequencies between the two systems is worthwhile. The identified natural frequencies for the second beam are all shifted down compared to those of the first. A reasonable explanation can be found in the different boundary conditions: the shaker, although suspended to the loading machine frame, represented a considerable mass attached to the tested specimen, which biased the identification results in a significant manner. 

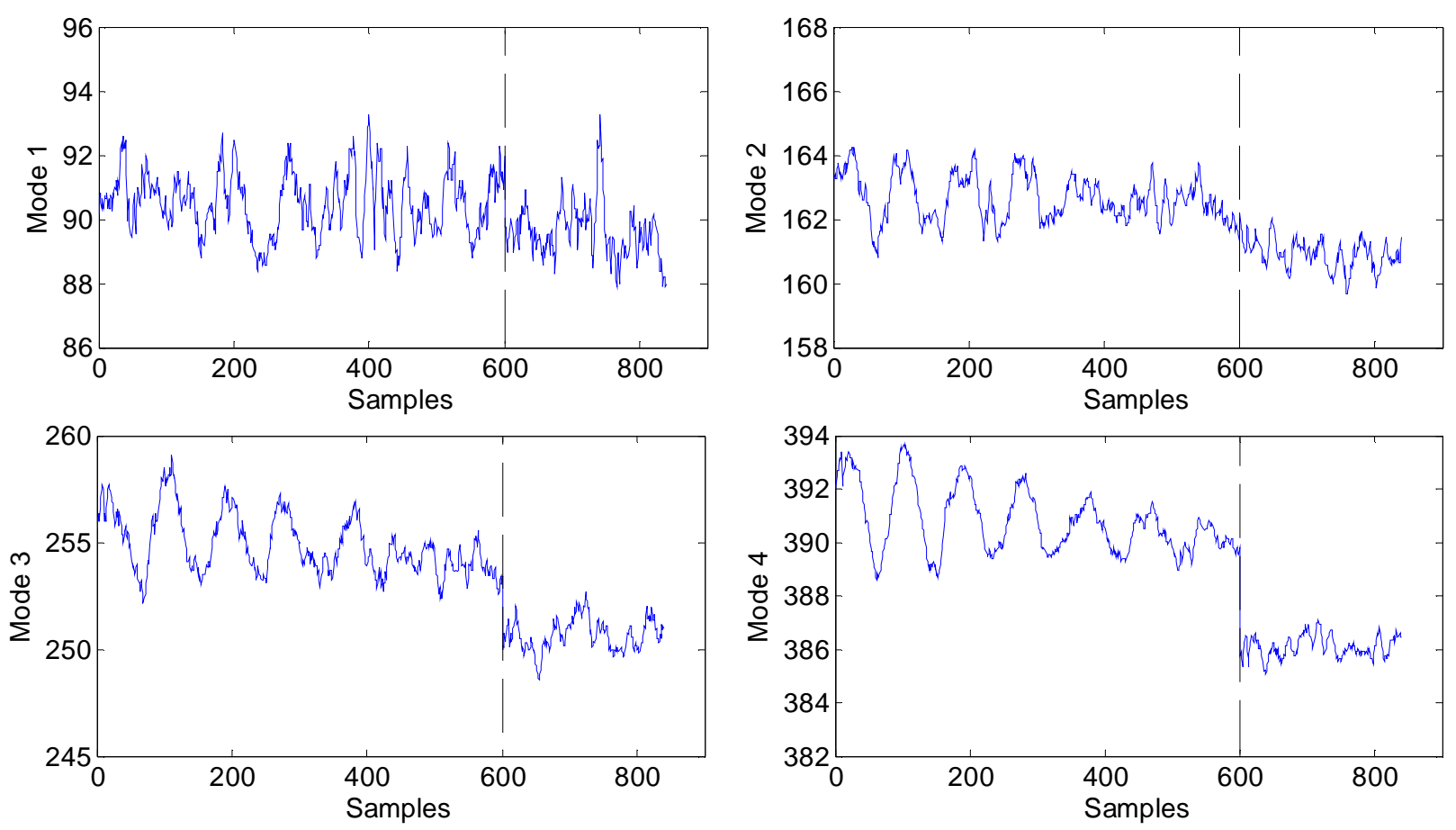

Figure 9: Evolution of the natural frequencies $[\mathrm{Hz}]$ throughout the tests carried out on the non-stationary system: damage introduced by saw-cut after the $600^{\text {th }}$ sample.

\section{Damage assessment under varying operational conditions}

\subsection{Different approaches to damage assessment of time-varying systems}

We have previously stressed the importance of the selection of damage-sensitive features and the crucial role played by the changes of environmental and operational conditions in masking damage events. The efforts of researchers have recently focused on the development of different approaches to damage assessment that take into account the environmental/operational issue in SHM. In this section a brief review of the available methods to deal with this problem is provided and some distinctions are made to circumscribe the case study here presented. First, for some approaches, the availability of measurements of the varying parameters (e.g., temperature, humidity, wind, traffic loading, etc.) is the main discriminant. If this is the case, different kinds of regression and interpolation analyses can be carried out to explicitly model the relationship between damage symptoms and the external variables [23].

Alternatively, other approaches attempt to subdivide the healthy reference state into a set of subdomains related to different environmental and operating conditions. Therefore, a comparison to detect damage is carried out between two states of the system (i.e., the reference state and that under investigation) that share the same environmental and operational conditions. Damage is detected when the 
structural response does not match with any reference state. However, the detection of an unexpected state, such as a damage symptom, presents two weak points: the difficulty in measuring all the influent environmental and operational parameters and, as a consequence, the impossibility to exhaustively define a priori all the reference states the structure may experience throughout its life-cycle. Thus, the reliability of the assessment is affected by a considerable risk of false-positive detection whenever a new state of the system not related to damage is encountered.

Sometimes direct measurements are not always practical and, often, the variability of the structural response depends upon so many factors that the definition of an explicit relation with the damage features becomes unfeasible. When direct measurements of environmental and operational conditions are not available, the detection heads towards the identification of changes in the extracted features that are "orthogonal" to the changes caused by the environmental and operational variations. This can be achieved by selecting features that are mainly sensitive to damage but insensitive or at least not very sensitive to the system variability and its environment. An example in this sense is the adoption of modal filters such as damage features, proposed by Deraemaeker and Preumont in [24], and the recognition of spurious peaks in their outputs for the detection.

Alternatively, an implicit modelling of the relationship between the environmental and operational variables and the damage sensitive features can be pursued without measuring the former factors. The features are projected in the subspace orthogonal to the one identified as the subspace wherein lie the external effects. Then, techniques such as factor analysis [25-26] and decomposition methods such as Principal Components Analysis (PCA) [27-28], Proper Orthogonal Decomposition (POD) [29-30] and Singular Value Decomposition (SVD) [31-33] can be employed to get rid of the environmental and operational effects.

Finally, some innovative approaches have been proposed to deal with the variability of environmental and operational conditions. In [34], Surace and Worden propose a negative selection algorithm inspired by the human immune system to detect novelties in structures subject to varying operational and environmental conditions. Cointegration is a promising analysis tool imported from the field of econometrics. It is developed in [35] in a nonlinear formulation, as to remove the dependency of the 
structural response on the environmental/operational influences, and to create a variable capable of maintaining its sensitivity to damage.

Two damage detection techniques are presented in the following to demonstrate the lack of accuracy of methods which disregard the variability of features related to external factors, and further to provide a reliable tool to deal with this problem. The results of the modal identification carried out on the timevarying system are adopted at this point.

\subsection{Reference-based damage detection}

A reference-based damage detection method is first applied in order to investigate the masking effects of the time-variant loading conditions on the damage features. The identified natural frequencies of the first four modes of the beam are selected to play this role. Here damage detection is conceived of as a novelty detection problem, which entails only the availability of data from the undamaged state of the structure, with no a priori information about the damaged states required. Indication of the damage occurrence is provided by a measure of the discordance of a candidate observation from the data set which represents the normal condition of the system. An observation which appears inconsistent with the data used to represent the baseline is considered as generated by a different state of the system and thus labelled as an outlier, i.e., a novelty. This process of outliers recognition is named Outlier Analysis.

The measure of discrepancy between two sets of data is the Mahalanobis squared distance, which suitably copes with the comparison of multivariate observations. The mapping between a candidate

observation $\left\{x_{c}\right\}$, defined as the set of damage sensitive features (i.e., the natural frequencies identified at each time-step), and the scalar damage index $D_{c}$ is provided by the following equation:

$$
D_{c}=\left(\left\{x_{c}\right\}-\{\bar{x}\}\right)^{T}[S]^{-1}\left(\left\{x_{c}\right\}-\{\bar{x}\}\right),
$$

where $\{\bar{x}\}$ and $[S]$ are respectively the mean vector and the covariance matrix of the reference data set. A Monte Carlo approach based on extreme value statistics is used to compute the threshold value that discriminates the candidate observations as inliers or outliers. The threshold value is extracted from a uniformly-distributed population with the same dimensions of the data set assumed to be the reference, i.e., the same number of features and observations. A confidence limit percentage of $1 \%$ is set to define 
the exceeding threshold over a large number of trials. When the Mahalanobis square distance of a candidate observation exceeds the threshold, a novelty is detected and a change in the system is inferred. For more details about the outlier analysis and its application to damage detection, the reader is referred to [36].

In this approach to damage detection, the reference set corresponds to a well-defined data set that is assumed as representative of the baseline of the system. The bounds of the reference set are arbitrarily established and they assume a crucial role, since the threshold value depends upon the size of the normal data. Moreover, all new observations are processed as candidate outliers according to Eq. (13), where the mean vector and the covariance matrix still depend upon the choice of the reference set.

Fig. 10 shows the result of the outlier analysis carried out on the first four identified frequencies of the quasi-stationary system. Up to the first 300 samples, the outcome is globally under the threshold (horizontal dashed line), which provides the indication of a healthy state of the structure. Very few normal observations exceeding the threshold (blue points circled in red) are erroneously classified as outliers. However, most of the damaged samples are below the threshold and circled in blue to signal the false-negative misclassifications. We can conclude that the accuracy of the outlier analysis is considerably affected by the masking effect of varying loading conditions.

\subsection{Reference-free damage detection}

The strong dependency of the damage detection technique on the selected reference set, as previously highlighted in the case of outlier analysis, represents a drawback for its application to a realistic SHM system. On the contrary, the methods based on the decomposition of the observations matrix have a powerful advantage from this point of view. These do not necessarily require a subjective definition of the reference set. 


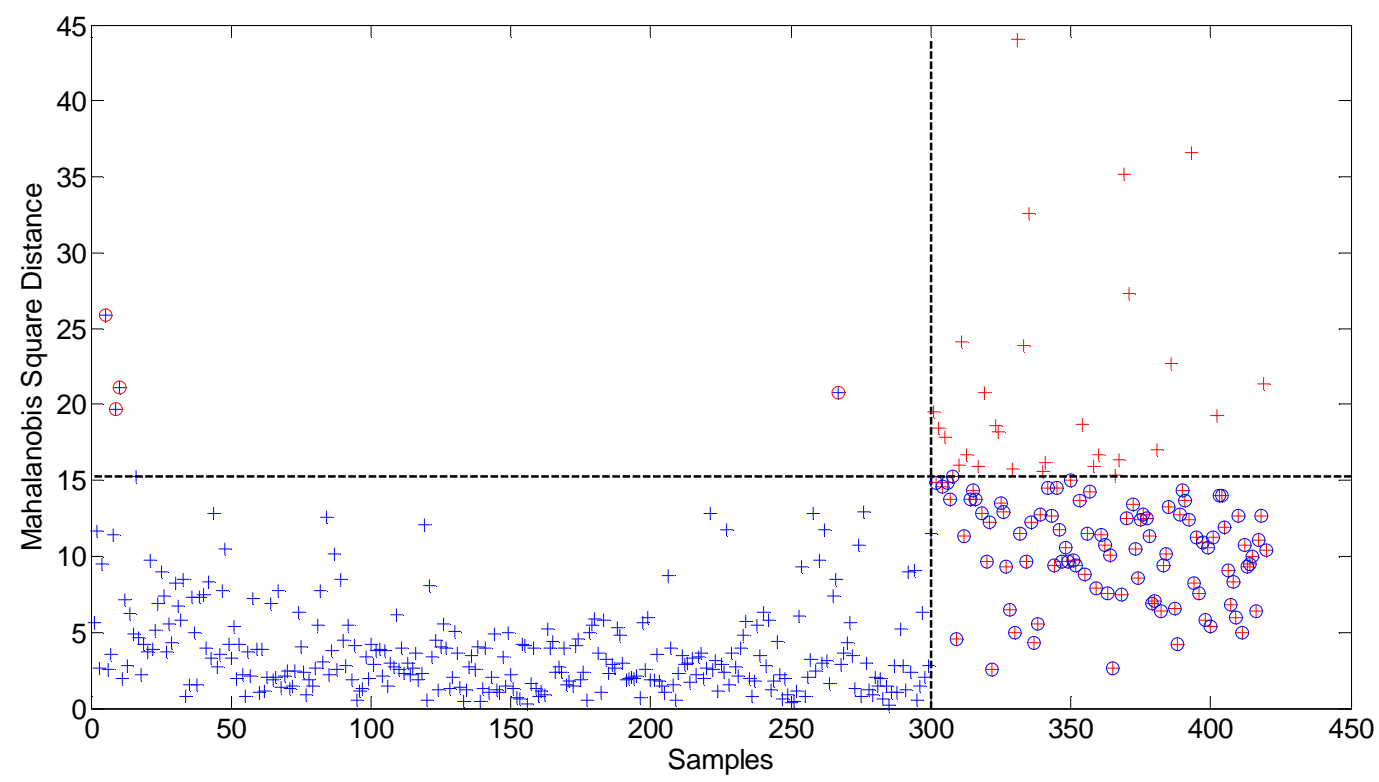

Figure 10: Outlier analysis result for the quasi-stationary system: healthy state is marked by blue crosses and the damaged one by red crosses. Red and blue circles detect false-positive and false-negative misclassifications.

A tight connection between different decompositions methods (namely PCA, POD and SVD) exists and is well-clarified in [29]. Analogously to modal analysis, and more generally to eigen-problems, decomposition methods aim at uncoupling the spatial and temporal content of the dynamic response of the system. Given an observations matrix $A$ composed by $m$ observations (rows) of $n$-variate features (columns), the SVD performs the decomposition:

$$
A=U \Sigma V^{T},
$$

where $U$ and $V$ are orthogonal matrices ( $m \times n$ and $n \times n$, respectively) and $\Sigma$ is a diagonal matrix ( $n \times \mathrm{x}$ n). $U$ and $V$ are commonly respectively referred to as left-hand and right-hand eigenvectors, or as singular vectors matrices; while $\Sigma$ is the eigenvalues or singular values matrix. The singular values are normally arranged in decreasing order and represent the extent of variability of the data projected in the reference space defined by the singular vectors. The link between SVD and the eigen-decomposition is proved by the correspondence between the singular values and vectors, and the eigenvalues and eigenvectors, derived from the matrix $\mathrm{AA}^{\mathrm{T}}$. Moreover, each singular value corresponds to a single basis function represented by each of the columns of the V matrix. Eq. (14) can be re-written as the product of two sets of functions: a temporal and a spatial one:

$$
A=Q V^{T}=\sum_{k=1}^{m} q_{k} v_{k}^{T},
$$


where the matrix $Q$ is obtained multiplying the matrices $U$ and $\Sigma$ and its columns $q_{k}$ are the functions that describe the temporal variation of the measurements. The $v_{k}$ are the basis functions responsible for the spatial variation of the measurements. In [37], the authors presented sufficient conditions on the response sampling, in order to retrieve the modal characteristics from the measured after-shock responses with a prescribed accuracy.

A reduced realization of the time-varying system can be computed by setting to zero the singular values related to undesired sources of variability. A reduced order singular values matrix $\Sigma_{\text {red }}$ is established and its adoption in (14) enables the uncounted variations to be filtered out. A reasonable choice when dealing with time-varying systems is to discard the main source of variability, which blurs the structural response thus preventing an effective assessment, in order to magnify more local and symptomatic events.

This specific interpretation of SVD is here exploited to detect abrupt changes of the selected damagesensitive features and use these as reliable indications of damage occurrence in varying environmental conditions. In the present case study, the first singular value is seen to carry $99.8 \%$ of the measurement variability, which can be reasonably ascribed to the response to time-varying loading. The remaining $0.2 \%$ is explained by the last three singular values, equal to $7.3,6.8$ and 4.1 , respectively. These can be considered substantially equivalent when compared to the value of 11847 obtained for the first.

To discard, or at least reduce, the effect of the varying stress, the reduced matrix $\Sigma_{\text {red }}$ is computed by setting the first diagonal element to zero. The result for the first system is shown in Fig. 11, where the monitored natural frequencies are represented according to the reduced order realization. It is worthwhile to notice that the exclusion of the first singular value, which carries most of the "energy" in the system, leads to the loss of the physical meaning of the measurements, whose values are now comparable and non-positive. The variations referring to the loading alterations are still identifiable but significantly reduced compared to those observed in Fig. 3. The damage occurrence is unequivocally detectable, as sudden changes for the last two features are amplified by the reduced order decomposition. Furthermore, the first feature seems to be insensitive to damage and to follow a similar trend to the time-varying loading. For the second one, a discontinuity is still present but partially masked by the significant load alterations. The damage sensitivity of the features mainly depends on the damage location, on the 
geometry, and on the boundary conditions of the structure. SVD amplifies the discontinuities introduced by local damage in some features even if these are hidden in the non-stationarity of data.
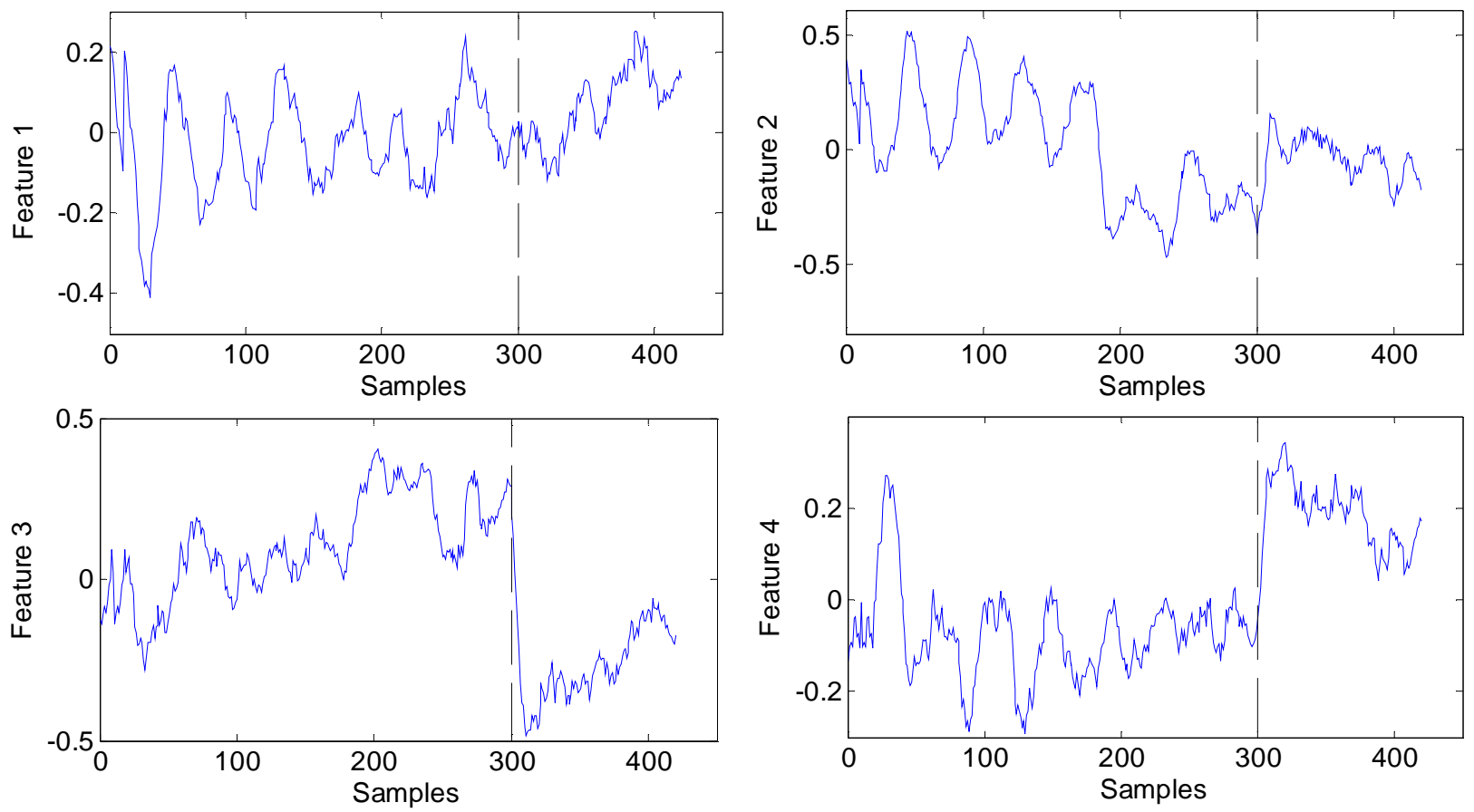

Figure 11: Damage features after reduced order realization through SVD: damage occurrence identifiable at the $300^{\text {th }}$ observation as trend discontinuity.

\section{Conclusions}

The effects of time-varying loads on the modal identification and damage assessment of beam/cablelike structures have been investigated. Spectral techniques based on Fourier Transforms have been successfully applied to extract modal parameters from quasi and non-stationary data. The implemented curve-fitting procedure proved to be an effective means to free the method from its dependency on the FFT frequency resolution. Computational simplicity and rapidity are the main advantages of these techniques which have been seen to accurately deal with slightly non-linear time-varying systems. For this purpose, wavelet transform-based techniques have been used to assess the linearity of the system and justify the adoption of the aforementioned techniques.

The presented SVD-based assessment method provides a reliable solution for the early detection of damage events in pre-stressed beam/cable-like structures subjected to changes of loading conditions. $A$ priori, the proposed methodology could be extended to the damage assessment of those structures that show a non-stationary behaviour related to changes of environmental and operational conditions. The 
SVD shows the ability to divorce the effects of exogenous events (long-term trend) from those related to damage. The latter appear as sudden "discontinuities" in the trend of features when a reduced order realization of the time-varying system is performed after the singular values decomposition. Limited to the results of the case study presented in this work, the SVD outperformed the OA, giving an important indication about the lack of accuracy of reference-based methods when these are applied to the damage detection of non-stationary systems. Moreover, a further advantage of the proposed methodology consists in its independence from a subjective definition of the reference set.

However, further investigations are required. These concern the selection of damage features and, more particularly, the definition of criteria to select the most damage-sensitive set. Indeed, the efficiency of the technique was seen to be dependent on the set of available natural frequencies considered in the decomposition. A more systematic study is required on the relation between physical (e.g., natural frequencies) and mathematical features (e.g., singular vectors derived from SVD). Further works are currently ongoing to evaluate the capability of the SVD to identify damage location by using modal shapes as features.

\section{References}

[1] C. Boller, F.-K. Chang, Y. Fujino, Encyclopedia of Structural Health Monitoring, John Wiley and Sons, 2009.

[2] J.M. Ko, Y.Q. Ni, Technological developments in structural health monitoring of large-scale bridges, Engineering Structures 27 (2005) 1715-1725.

[3] H. Sohn, Effects of environmental and operational variability on structural health monitoring, Phil. Trans. R. Society A 365 (2007) 539-560.

[4] N.M.M. Maia, J.M.M. Silva, Theoretical and Experimental Modal Analysis, John Wiley and Sons, 1997.

[5] B. Peeters, G. De Roeck, Reference-based stochastic subspace identification for output-only modal analysis, Mechanical Systems and Signal Processing 13 (6) (1999) 477-489.

[6] S. Mallat, A Wavelet Tour of Signal Processing, Academic Press, (1998).

[7] T.P. Le, P. Argoul, Continuous wavelet transform for modal identification using free decay response, Journal of Sound and Vibration, 277 (2004) 73-100.

[8] W.J. Staszewski, Structural and mechanical damage detection using wavelets, The Shock and Vibration Digest, 30 (6) (1998) 457-472. 
[9] S. Marchesiello, S. Bedaoui, L. Garibaldi, P. Argoul, Time-dependent identification of a bridge-like structure with crossing loads, Mechanical Systems and Signal Processing, 23 (2009) 2019-2028.

[10] N.E. Huang, Z. Shen, S.R. Long, M.C. Wu, H.H. Shih, Q. Zheng, N. C. Yen, C.C. Tung, H.H. Liu, The empirical mode decomposition and the Hilbert spectrum for nonlinear and non-stationary time series analysis, Proceedings of the Royal Society of London, 454 (1998) 903-995.

[11] J.N. Yang, Y. Lei, S. Pan, N. Huang, System identification of linear structures based on Hilbert-Huang spectral analysis. Part 1: normal modes, Earthquake Eng. and Structural Dynamics, 32 (2003) 1443-1467.

[12] Y.L. Xu, J. Chen, Structural damage detection using empirical mode decomposition: Experimental investigation, Journal of Engineering Mechanics, 130 (11) (2004), 1279-1288.

[13] C.R. Farrar, W.E. Baker, T.M. Bell, K.M. Cone, T.W. Darling, T.A. Duffey, A. Eklund, A. Migliori, Dynamic characterization and damage detection in the I-40 bridge over the Rio Grande, Los Alamos National Laboratory Report: LA-12767-MS.

[14] J. S. Bendat, A. G. Piersol, Engineering Application of Correlation and Spectral Analysis, Wiley-Interscience, (1980).

[15] R. Ceravolo, Use of instantaneous estimators for the evaluation of structural damping, Journal of Sound and Vibration, 274 (2004), 385-401.

[16] P. Argoul, T-P. Le, Instantaneous indicators of structural behaviour based on the continuous Cauchy wavelet transform, Mechanical Systems and Signal Processing, 17 (2003) 243-250.

[17] S. Erlicher, P. Argoul, Modal identification of linear non-proportionally damped systems by wavelet transform, Mechanical Systems and Signal Processing, 21(3) (2007) 1386-1421.

[18] P. Argoul, Quelques réflexions sur l'identification de paramètres en dynamique des matériaux et des structures. Mémoire d'Habilitation à Diriger des Recherches (in French), (2004).

[19] V. Perier, L. Dieng, L. Gaillet, C. Tessier, S. Fouvry, Fretting-fatigue behaviour of bridge engineering cables in a solution of sodium chloride, Wear 267 (1-4) (2009), 308-314.

[20] M. Matsumoto, N. Shiraishi, H. Shirato, Rain-wind induced vibration of cables of cable-stayed bridges, Journal of Wind Engineering and Industrial Aerodynamics, 43 (1) (1992), 2011-2022.

[21] D. Siegert, L. Dieng, M. Goursat, F. Toutlemonde, Frequency monitoring of stay-cables, In Proc. of the XXV International Modal Analysis Conference, Orlando, USA, 2007

[22] T-T-H. Le, Contribution à la détection, à la localisation et au suivi par méthode d'évaluation dynamique, de l'endommagement des câbles de génie civil, in French, Université Paris Est, 2011-2013. 
[23] B. Peeters, G. De Roeck, One year monitoring of the Z24 bridge: environmental influences versus damage effects, Proc. IMAC-XVIII San Antonio, TX, 1570-1579.

[24] A. Deraemaeker, A. Preumont, Vibration-based damage detection using large array sensors and spatial filters, Mechanical Systems and Signal Processing, 20 (2006) 1615-1630.

[25] J. Kullaa, Structural health monitoring under variable environmental or operational conditions, Proc. EWSHM-II Munich, Germany (2004) 1262-1269.

[26] A. Deraemaeker, E. Reynders, G. De Roeck, J. Kullaa, Vibration-based structural health monitoring using output-only measurements under changing environment, Mechanical Systems and Signal Processing, 22 (2008) 34-56.

[27] G. Manson, Identifying damage sensitive, environment insensitive features for damage detection, Proc. Third International Conference on Identification in Engineering Systems, Swansea, UK (2002).

[28] A. Yan, G. Kerschen, P.D. Boe, J. Golinval, Structural damage diagnosis under varying environmental conditions - part I: a linear analysis, Mechanical Systems and Signal Processing, 19 (4) (2005) 847-864.

[29] F. Lanata, A. Del Grosso, Damage detection and localization for continuous static monitoring of structures using a proper orthogonal decomposition of signals, Smart Materials and Structures, 15 (2006) 1811-1829.

[30] U. Galvanetto, G. Violaris, Numerical investigations of a new damage detection method based on proper orthogonal decomposition, Mechanical Systems and Signal Processing, 21 (2007) 1346-1361.

[31] C. Shane, R. Jha, Proper orthogonal decomposition based algorithm for detecting damage location and severity in composite beams, Mechanical Systems and Signal Processing, 25 (2011) 1062-1072.

[32] R. Ruotolo, C. Surace, Using SVD to detect damage in structures with different operational conditions, Journal of Sound and Vibration, 226 (3) (1999).

[33] S. Vanlanduit, E. Parloo, B. Cauberghe, P. Guillaume, P. Verboven, A robust singular value decomposition for damage detection under changing operation conditions and structural uncertainties, Journal of Sound and Vibration, 284 (2005) 1033-1050.

[34] C. Surace, K. Worden, Novelty detection in a changing environment: A negative selection approach, Mechanical Systems and Signal Processing, 24 (2010) 1114-1128.

[35] E.J. Cross, K. Worden, Approaches to nonlinear cointegration with a view towards applications in SHM, Journal of Physics: Conference Series, 305 (2011).

[36] K. Worden, G. Manson, N.R.J. Fieller, Damage detection using outlier analysis, Journal of Sound and Vibration, 229 (3) (2000), 647-667. 
[37] R. Elias, N. Point, J. Bodgi, P. Argoul, How to retrieve the normal modes using the POD, Proc. XVIII symposium Vibrations chocs et Bruit \& ASTELAB, EDF CLAMART : France (2012). 